\title{
Effect of Age on Effectiveness of Vonoprazan in Triple Therapy for Helicobacter pylori Eradication
}

\author{
Maho Kusunoki ${ }^{1,2}$, Mika Yuki ${ }^{1}$, Hitomi Ishitobi ${ }^{1}$, Yoshiya Kobayashi ${ }^{1}$, Makoto Nagaoka ${ }^{1}$, \\ Yoshiko Takahashi ${ }^{1}$, Nobuhiko Fukuba ${ }^{1}$, Yoshinori Komazawa ${ }^{1}$, \\ Toshihiro Shizuku ${ }^{1}$ and Yoshikazu Kinoshita ${ }^{2}$
}

\begin{abstract}
:
Objective We evaluated the efficacy of vonoprazan-based eradication therapy for Helicobacter pylori $(H$. pylori), including the effects of age, gender, and grade of atrophy in comparison to proton pump inhibitorbased therapy.

Method We retrospectively reviewed the records of 1,172 patients who received first-line triple therapy with amoxicillin, clarithromycin, and vonoprazan or a proton pump inhibitor (PPI) for H. pylori eradication, as well as 157 patients treated with second-line therapy consisting of amoxicillin, metronidazole, and vonoprazan or a PPI.

Results The eradication rate of all cases treated with first-line triple therapy was $86.9 \%(1,019 / 1,172)$, while that in those treated with vonoprazan-based therapy was $92.5 \%$ (384/415). Our analysis showed that the use of vonoprazan resulted in a significantly improved success rate of first-line eradication therapy in comparison to proton pump inhibitor-based therapy [odds ratio (OR), 2.36; 95\% confidence interval (CI) 1.55 to 3.56]. The superiority of vonoprazan was remarkable in non-elderly patients, while its effect was unclear in elderly patients. When used as second-line eradication therapy, the advantage of vonoprazan over PPI administration was not clear.

Conclusion The inclusion of vonoprazan increased the success rate of first-line eradication therapy; however, the advantage was reduced with aging and remained unclear in elderly patients.
\end{abstract}

Key words: Helicobacter pylori, eradication, potassium-competitive acid blockers, elderly, atrophic gastritis, second-line therapy

(Intern Med 58: 1549-1555, 2019)

(DOI: 10.2169/internalmedicine.2233-18)

\section{Introduction}

There have been dramatic changes in the options for the eradication of Helicobacter pylori (H. pylori). In 2013, eradication therapy for gastritis caused by $H$. pylori infection was approved by the national health insurance system of Japan, resulting in an increased number of cases. Subsequently, in 2015, vonoprazan, a novel potassium-competitive acid blocker, was released and approved for the eradication of $H$. pylori infection.
A phase III randomized double-blind study showed that vonoprazan was not inferior to lansoprazole in first-line eradication therapy when either was used in combination with amoxicillin and clarithromycin, while a high rate of eradication was also shown in subjects who underwent vonoprazan-based second-line therapy with amoxicillin and metronidazole (1). Subsequently, a higher rate of $H$. pylori eradication was reported with vonoprazan-based therapy in comparison to proton pump inhibitor-based therapy (2). On the other hand, when used in second-line eradication therapy, the effectiveness of vonoprazan over the administration

${ }^{1}$ Department of Internal Medicine, Izumo City General Medical Center, Japan and ${ }^{2}$ Department of Gastroenterology and Hepatology, Shimane University School of Medicine, Japan

Received: October 5, 2018; Accepted: November 14, 2018; Advance Publication by J-STAGE: February 1, 2019

Correspondence to Dr. Maho Kusunoki, kusunokiemail@gmail.com 
of a proton pump inhibitor (PPI) has not been demonstrated (3-5). In addition, it remains unclear whether elderly patients require strong acid suppression drugs such as vonoprazan for the eradication of $H$. pylori, because gastric acid secretion decreases with age (6), which is a major issue throughout the world because of aging populations (7).

In the present study, we evaluated the rates of successful eradication in patients receiving first- and second-line therapy in order to compare the efficacy of vonoprazan to PPI administration, and also examined the effects of age.

\section{Materials and Methods}

\section{Study design}

In this retrospective open-label single-center study we reviewed the findings from patients who underwent $H$. pylori eradication therapy in a standard 7-day protocol. The eradication rates according to age, gender, grade of gastric mucosal atrophy, and acid suppression drugs were determined. The gastric mucosal atrophy grade was included in the analysis, as it is an indicator of acid secretion, which decreases with the progression of atrophic gastritis (6). The study was conducted in accordance with the Declaration of Helsinki and was approved by the ethics committee of Izumo-City General Medical Center.

\section{Patients}

Patients who underwent $H$. pylori eradication therapy at Izumo-City General Medical Center from January 2013 to April 2017 were enrolled in this retrospective study. We confirmed the success or failure of eradication in all cases during the study period. Second-line eradication therapy was only performed in cases in which first-line therapy failed.

\section{The diagnosis and eradication of H. pylori}

H. pylori infection was diagnosed based on the $H$. pylori $\mathrm{IgG} \mathrm{Ab}$ titer [ $\geq 10 \mathrm{U} / \mathrm{mL}$ (plate E 'Eiken' $H$. pylori $\mathrm{Ab}$ )]. $H$. pylori eradication was evaluated using a $13 \mathrm{C}$-urea breath test at least 4 weeks after the end of therapy.

For treatment, each patient received the standard eradication therapy protocol used in Japan. PPI-based first-line therapy contained amoxicillin $(750 \mathrm{mg}$ ) and clarithromycin (200 or $400 \mathrm{mg}$ ) with a PPI twice daily for 1 week (PAC regimen). Vonoprazan-based first-line therapy, which we began to prescribe in March 2015, contained amoxicillin (750 $\mathrm{mg}$ ) and clarithromycin (200 or $400 \mathrm{mg}$ ) with vonoprazan (20 mg) (VAC regimen). For second-line therapy we prescribed metronidazole $(250 \mathrm{mg})$ instead of clarithromycin. PPI-based second-line therapy contained a PPI, amoxicillin, and metronidazole (PAM), and vonoprazan-based secondline therapy contained vonoprazan, amoxicillin and metronidazole (VAM). The PPIs administered to the present patients included esomeprazole (20 mg), lansoprazole (30 mg), and rabeprazole (10 mg). From January 2013 to February 2015, PPI based-therapy was exclusively prescribed, then from
March 2015 to April 2017 the attending physician chose between vonoprazan- and PPI-based therapy depending on their individual treatment policy.

\section{Assessment of gastric mucosal atrophy}

Gastric mucosal atrophy was diagnosed based on the Kimura-Takemoto classification using esophagogastroduodenoscopy findings (8). We classified patients into mild and severe atrophy groups. Mild atrophy was defined by the absence of atrophy or the presence of an atrophic border on the lesser curve, the same as the closed type in the KimuraTakemoto classification. Severe atrophy was defined by a greater spread, the same as the open type in the KimuraTakemoto classification.

\section{Statistical analysis}

Continuous variables were analyzed using the MannWhitney test. Binary variables were analyzed using a chisquared test or Fisher's exact test. A Steel-Dwass test was performed to compare multiple groups. A multivariate logistic regression analysis was performed to determine odds ratios (ORs) and 95\% confidence intervals (95\% CIs). P values of $<0.05$ were considered to indicate statistical significance. Statistical analyses were conducted using the IBM SPSS (version 22.0) or Bellcurve for Excel (version 2.14) software programs.

\section{Results}

\section{H. pylori eradication}

A total of 1,201 patients completed $H$. pylori eradication treatment, with first-line therapy administered to 1,172 and second-line therapy administered to 157 . The baseline characteristics of the patients are shown in Table 1.

The eradication rate for all patients was $96.8 \%(1,162 /$ $1,201)$, while the rates for first-line therapy and second-line therapy cases were $86.9 \%(1,019 / 1,172)$ and $91.1 \%(143 /$ 157), respectively (Table 1 ). When the patients were divided by age, the success rate of first-line eradication therapy in patients of $\leq 39$ years of age was significantly lower in comparison to that in patients of 40-49 ( $\mathrm{p}=0.012), 50-59(\mathrm{p}=$ $0.003)$, and 60-69 ( $\mathrm{p}=0.009)$ years of age. When the patients were divided according to use of acid suppression drugs, the rate of eradication with vonoprazan was significantly higher than that with lansoprazole $(\mathrm{p}=0.001)$ or rabeprazole $(\mathrm{p}<$ $0.001)$. When the patients were divided according to gender and grade of gastric mucosal atrophy, there were no significant differences in the success rate of first-line eradication. In the multivariate logistic regression analysis, with age, gender, gastric mucosal atrophy grade, and acid suppression drugs employed as variables, only vonoprazan had a significant impact on first-line eradication success (OR, 2.36; 95\% CI 1.55 to 3.56) (Table 2). None of those variables had a significant effect on the success of second-line therapy. 
Table 1. Patient Baseline Characteristics and Rates of H. Pylori Eradication Based on Therapy.

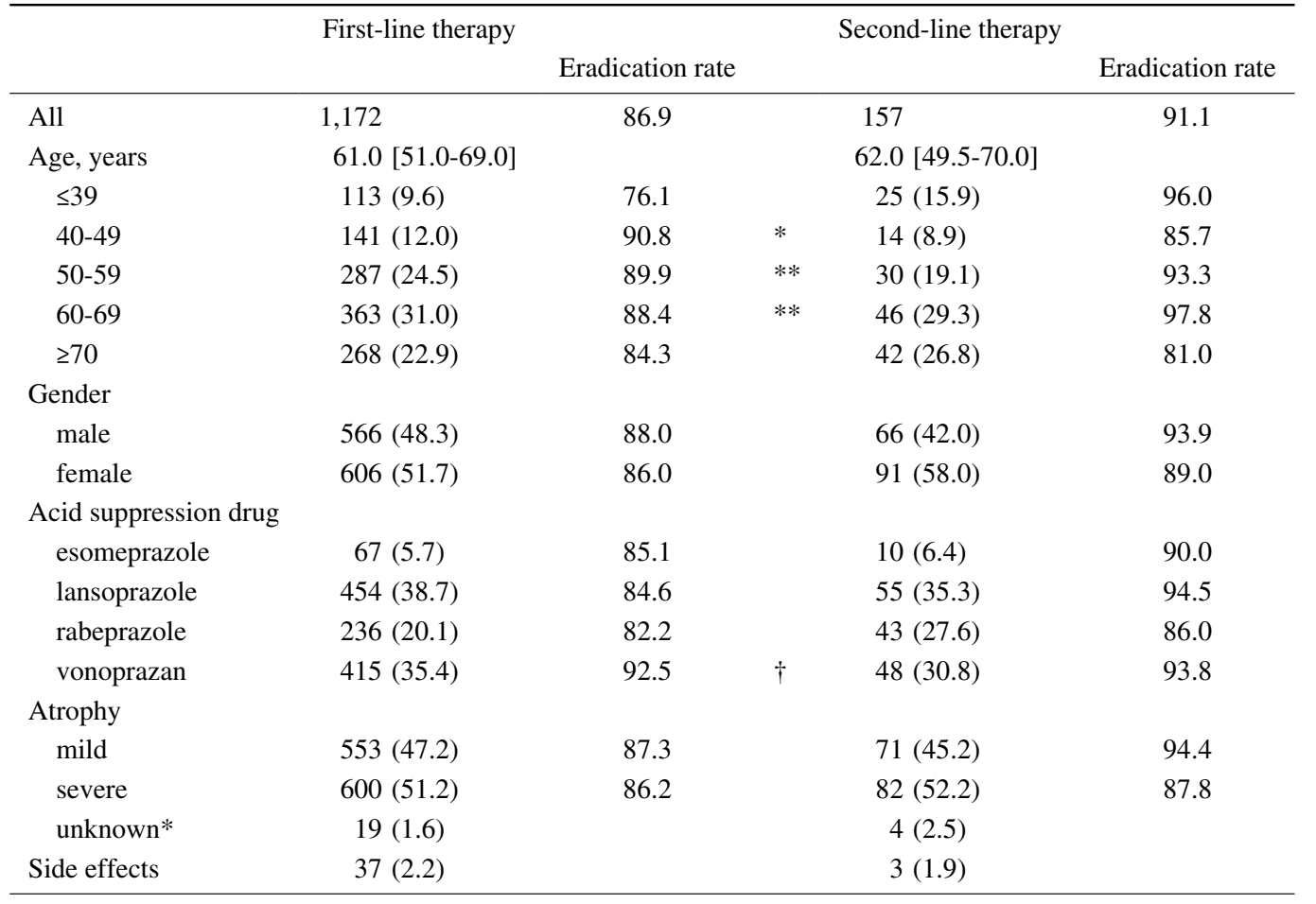

Values are shown as n (\%) or median [IQR]. *Including post-distal gastrectomy state.

Eradication therapy success shown as percentage.

$*$ p $<0.05$, vs. $\leq 39$ years; $* *$ p $<0.01$, vs. $\leq 39$ years; $\uparrow p<0.05$, vs. lansoprazole and rabeprazole (Steel-Dwass test).

\section{Comparison between vonoprazan and PPI}

The baseline characteristics of the patients who received vonoprazan and those who received a PPI are shown in Table 3. Regardless of gender and grade of atrophy, the VAC regimen was superior to the PAC regimen when used as first-line therapy (Table 3). On the other hand, neither regimen was superior in the older age groups (60-69, $\geq 70$ years), while the VAC regimen was superior in non-elderly patients.

In the univariate analysis, age, gender, and grade of atrophy had no significant effect on eradication success with the VAC regimen, while the eradication rate in patients of $\leq 39$ years of age was significantly lower in comparison to the rates in older age groups that received the PAC regimen (Table 3). A multiple logistic regression analysis that included age, gender, and grade of atrophy as independent variables showed that age had a slightly negative effect in patients that received the VAC regimen $(\mathrm{OR}, 0.95 ; 95 \% \mathrm{CI} 0.92$ to 0.98 ) and a slightly positive effect in those that received the PAC regimen (OR, 1.02; 95\%CI 1.00 to 1.04) (Table 2). As for second-line eradication therapy, analyses performed according to the above-mentioned methods revealed no significant differences.

\section{Discussion}

In the present study population, the success rate of firstline eradication therapy with the VAC regimen was signifi- cantly greater than that with the PAC regimen. However, there were no significant differences between those regimens when they were administered to elderly patients. In addition, there was no clear advantage of the VAM regimen when used as second-line therapy.

Seven-day PPI-based amoxycillin clarithromycin triple therapy was the standard first-line therapy for $H$. pylori eradication in Japan prior to the approval of vonoprazan. Both amoxicillin and clarithromycin require organism growth for their effectiveness. H. pylori organisms can grow in the periplasm at in the $\mathrm{pH}$ range of 6.0 to 8.0. The administration of a PPI increases the population of dividing organisms by elevating the gastric $\mathrm{pH}$, and thus synergizes with antibiotics (9). Insufficient acid suppression can cause eradication failure (10). Another serious problem with this regimen is a decreasing trend in the rate of eradication, which is related to increasing clarithromycin resistance $(11,12)$. It seems that the frequent administration of clarithromycin to patients with respiratory infections has contributed to a remarkable increase in the rate of resistance in Japan (13).

Vonoprazan, a novel potassium-competitive acid blocker, inhibits gastric $\mathrm{H}^{+}$and $\mathrm{K}^{+}$-ATPase like PPIs, but in a $\mathrm{K}^{+}$competitive and reversible manner, unlike PPIs. Acid activation is not required for vonoprazan to exert its acid secretion inhibiting effect and this drug exerts a more potent inhibitory effect on gastric acid secretion, and also has a stronger and longer lasting effect on elevated $\mathrm{pH}$ in gastric perfusate in comparison to lansoprazole (14). A randomized open- 


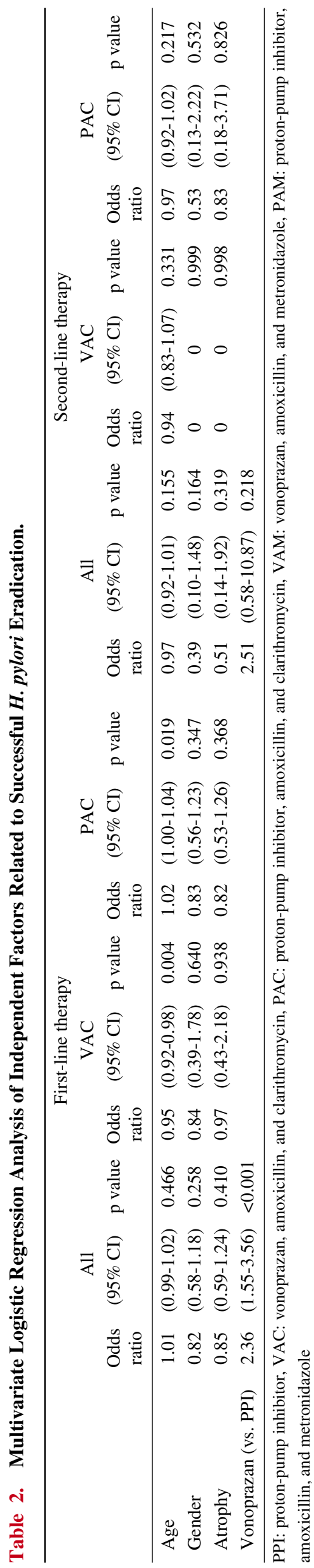

label crossover study also showed that vonoprazan has more rapid and sustained acid-inhibitory effects than esomeprazole and rabeprazole (15). Thus, the VAC regimen has a higher eradication rate than the PAC regimen. Moreover, post hoc analyses conducted in a phase III study indicated that the eradication rate in patients infected with clarithromycinresistant strains who received the VAC regimen was significantly higher than that in those who received the lansoprazole, amoxicillin and clarithromycin regimen. Additionally, the analysis revealed the superiority of the VAC regimen in patients with the extensive metabolizer genotype CYP2C19, an enzyme known to metabolize PPIs (1).

In the present investigation, the superiority of vonoprazanbased treatment to PPI-based treatment was limited to younger or middle-aged patients undergoing first-line therapy. The post hoc analysis findings in the phase III study showed that the superiority of VAC was not clear in individuals of $\geq 65$ years of age. Our results confirmed that the efficacy of the VAC regimen over the PAC regimen is conspicuous in patients of $\leq 39$ years of age, and declines with age, eventually becoming unclear in patients of $\geq 60$ years of age, which seems to be related to the fact that gastric acid secretion is abundant in younger individuals and decreases with aging (6). This suggests that elderly patients, who have decreased acid secretion, might not require strong acid suppressing drugs like vonoprazan for $H$. pylori eradication and that a PPI may be adequate. Furthermore, factors other than insufficient acid suppression may be more associated with eradication failure in elderly patients. In young patients, the PAC regimen was associated with a low eradication rate, which seems to be related to not only their abundant acid secretion but also a markedly increased prevalence of clarithromycin resistance in younger individuals (16). Thus, in both respects, the VAC regimen seems to be beneficial for young patients.

On the other hand, the VAC regimen was associated with a significantly higher eradication rate than the PAC regimen in patients with mild gastric mucosal atrophy, in which gastric acid secretion can be expected to be relatively maintained, and in those with severe mucosal atrophy, in which gastric acid secretion can be expected to be decreased (6). A possible reason is that the Kimura-Takemoto classification, in which the endoscopic atrophic border is the basis for diagnosis, does not exactly reflect histological mucosal atrophy or inflammation $(17,18)$. The prediction of acid secretion based on the Kimura-Takemoto classification may be more difficult because of a declining parietal cell function, which plays an important role in inhibiting acid secretion during $H$. pylori infection (19). As another potential explanation, a delay in the gastric emptying time due to advanced atrophy may deactivate a PPI administered in the form of an enteric-coated tablet (20). That is, the severity of gastric mucosal atrophy was not apparently associated with the outcomes of patients treated with the PAC regimen, because delayed gastric emptying as well as decreased acid secretion may affect eradication in cases of severe atrophy. On the 


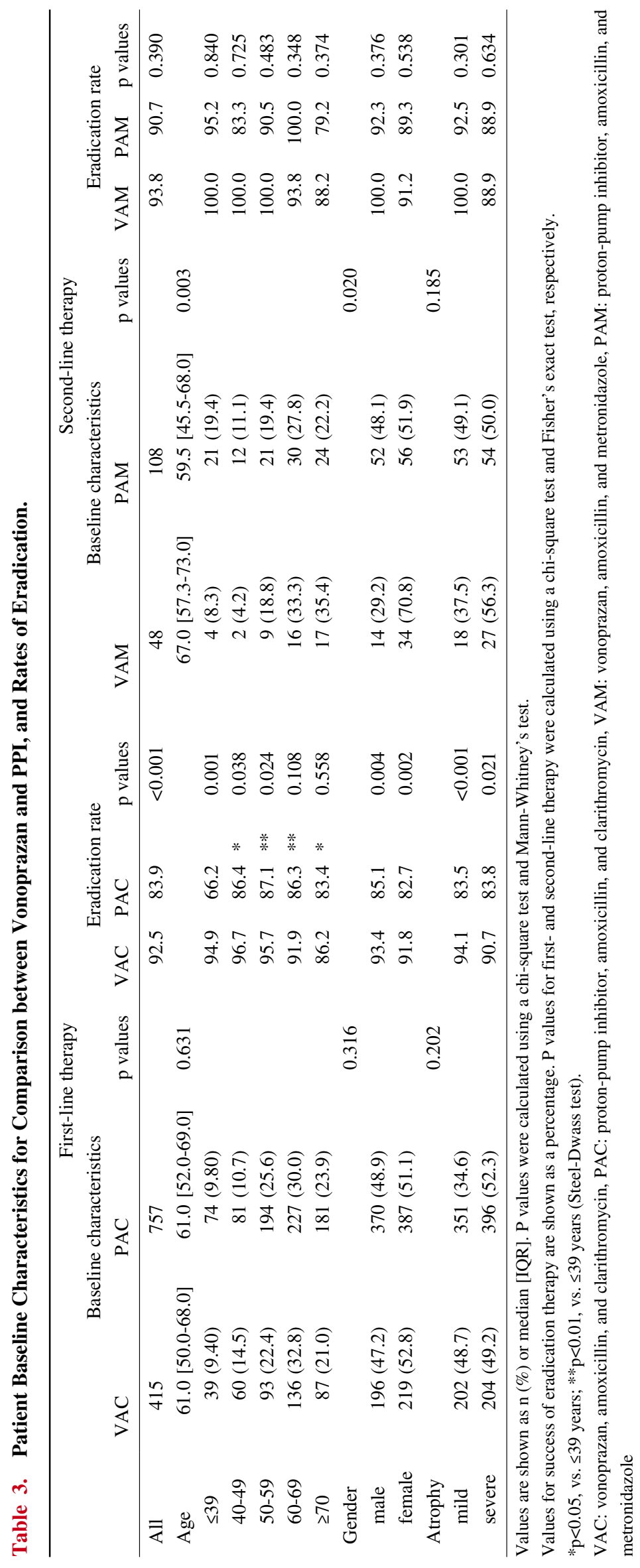

other hand, vonoprazan, which is not available in an enteric- gardless of the severity of gastric mucosal atrophy.

coated tablet form, may potently inhibit acid suppression re-

In the present study, the patients with severe gastric mu- 
cosal atrophy treated with first-line therapy were older than those with mild atrophy [median 65.0 years (interquartile range 58.0-72.0) vs. 55.0 years (44.0 to 64.0), $\mathrm{p}<0.001]$. Indeed, gastric mucosal atrophy extends with aging, resulting in decreased gastric acid secretion in $H$. pylori-positive patients (6). Furthermore, gastric acid secretion in elderly patients is often reduced by factors other than atrophic gastritis caused by $H$. pylori (21). Aging, which can be directly evaluated, seems to be an important factor in relation to gastric acid secretion. Our results suggest that patient age may be more useful for choosing an acid suppressing drug for $H$. pylori eradiation than endoscopic atrophy.

In the present study, the VAM regimen was not superior to the PAM regimen for second-line eradication therapy regardless of age. The main reason seems to be that the efficacy of metronidazole, which targets DNA, is independent of the stationary or growth phase distribution, as it is does not require acid suppression (22). In addition, it may have had effects on equality, as metronidazole has a lower rate of resistance than clarithromycin due to its limited use in the treatment of anaerobic and protozoan infections in Japan, despite the fact that increasing rates of metronidazole resistance have been reported in various regions of the world $(12,23,24)$. It seems that the role of acid suppression in second-line therapy is reduced in comparison to first-line therapy.

This study was associated with some limitations, including its retrospective single center design. We did not evaluate antibiotic resistance or the expression of CYP2C19, which are known to be major causes of eradication failure. Notably, it is recommended that clarithromycin resistance, which greatly affects eradication, is evaluated before starting eradication therapy. We diagnosed $H$. pylori infection based on the H. pylori $\operatorname{IgG} \mathrm{Ab}$ titer. This method is commonly used and is suitable for clinical practice even in our hospital where many patients come for medical checkups, as the procedure is only minimally invasive and the results can be obtained within 1 day. Furthermore, the registration periods of patients who received vonoprazan- and PPI-based therapy were different. Vonoprazan has been prescribed since March 2015 , following its release in the previous month. This resulted in a decrease in the number of PPI prescriptions. In this study, clarithromycin (200 or $400 \mathrm{mg}$ ) was administered in the first-line therapy regimen, although its impact on the present results was likely not significant (25). Based on these limitations, our results must be interpreted with care.

The present findings demonstrate that the efficacy of vonoprazan is superior to that of PPI treatment for first-line therapy, while there were no significant differences seen between them when used for second-line therapy. Furthermore, the superiority of vonoprazan in first-line therapy for elderly patients was not clear.

The authors state that they have no Conflict of Interest (COI).

\section{References}

1. Murakami K, Sakurai Y, Shiino M, Funao N, Nishimura A, Asaka M. Vonoprazan, a novel potassium-competitive acid blocker, as a component of first-line and second-line triple therapy for Helicobacter pylori eradication: a phase III, randomised, double-blind study. Gut 65: 1439-1446, 2016.

2. Jung YS, Kim EH, Park CH. Systematic review with metaanalysis: the efficacy of vonoprazan-based triple therapy on Helicobacter pylori eradication. Aliment Pharmacol Ther 46: 106-114, 2017.

3. Tsujimae M, Yamashita $\mathrm{H}$, Hashimura $\mathrm{H}$, et al. A comparative study of a new class of gastric acid suppressant agent named vonoparazan versus esomeprazole for the eradication of Helicobacter pylori. Digestion 94: 240-246, 2016.

4. Yamada S, Kawakami T, Nakatsugawa Y, et al. Usefulness of vonoprazan, a potassium ion-competitive acid blocker, for primary eradication of Helicobacter pylori. World J Gastrointest Pharmacol Ther 7: 550-555, 2016.

5. Sue S, Kuwashima $H$, Iwata $Y$, et al. The superiority of vonoprazan-based first-line triple therapy with clarithromycin: a prospective multicenter cohort study on Helicobacter pylori eradication. Intern Med 56: 1277-1285, 2017.

6. Haruma K, Kamada T, Kawaguchi H, et al. Effect of age and Helicobacter pylori on gastric acid secretion. J Gastroenterol Hepatol 15: 277-283, 2000.

7. United Nations, Department of Economic and Social Affairs, Population Division (2017). World Population Prospects: The 2017 Revision, Key Findings and Advance Tables. Working Paper No. $\mathrm{ESA} / \mathrm{P} / \mathrm{WP} / 248$.

8. Kimura K, Takemoto T. An endoscopic recognition of the atrophic border and its significance in chronic gastritis. Endoscopy 1: 8797, 1969.

9. Scott D, Weeks D, Melchers K, Sachs G. The life and death of Helicobacter pylori. Gut 43(suppl 1): S56-S60, 1998.

10. Villoria A, Garcia P, Calvet X, Gisbert JP, Vergara M. Metaanalysis: high-dose proton pump inhibitors vs. standard dose in triple therapy for Helicobacter pylori eradication. Aliment Pharmacol Ther 28: 868-877, 2008.

11. Lee JY, Kim N, Kim MS, et al. Factors affecting first-line triple therapy of Helicobacter pylori including CYP2C19 genotype and antibiotic resistance. Dig Dis Sci 59: 1235-1243, 2014.

12. Kobayashi I, Murakami K, Kato M, et al. Changing antimicrobial susceptibility epidemiology of Helicobacter pylori strains in Japan between 2002 and 2005. J Clin Microbiol 45: 4006-4010, 2007.

13. Perez Aldana L, Kato M, Nakagawa M, et al. The relationship between consumption of antimicrobial agents and the prevalence of primary Helicobacter pylori resistance. Helicobacter 7: 306-309, 2002.

14. Hori Y, Imanishi A, Matsukawa J, et al. 1-[5-(2-Fluorophenyl)-1-( pyridin-3-ylsulfonyl)-1H-pyrrol-3-yl]-N-methylmethanamine monofumarate (TAK-438), a novel and potent potassium-competitive acid blocker for the treatment of acid-related diseases. J Pharmacol Exp Ther 335: 231-238, 2010.

15. Sakurai Y, Mori Y, Okamoto H, et al. Acid-inhibitory effects of vonoprazan $20 \mathrm{mg}$ compared with esomeprazole $20 \mathrm{mg}$ or rabeprazole 10mg in healthy adult male subjects - a randomised openlabel cross-over study. Aliment Pharmacol Ther 42: 719-730, 2015.

16. Okamura $T$, Suga $T$, Nagaya $T$, et al. Antimicrobial resistance and characteristics of eradication therapy of Helicobacter pylori in Japan: a multi-generational comparison. Helicobacter 19: 214-220, 2014.

17. Nomura S, Ida K, Terao S, et al. Research Group for Establishment of Endoscopic Diagnosis of Chronic Gastritis Endoscopic diagnosis of gastric mucosal atrophy: multicenter prospective study. 
Dig Endosc 26: 709-719, 2014.

18. Nomura S, Terao S, Adachi K, et al. Endoscopic diagnosis of gastric mucosal activity and inflammation. Dig Endosc 25: 136-146, 2013.

19. Osawa $\mathrm{H}$, Kita $\mathrm{H}$, Ohnishi $\mathrm{H}$, et al. Helicobacter pylori eradication induces marked increase in $\mathrm{H}^{+} / \mathrm{K}^{+}$-adenosine triphosphatase expression without altering parietal cell number in human gastric mucosa. Gut 55: 152-157, 2006.

20. Tucci A, Poli L, Biasco G, et al. Helicobacter pylori infection and gastric function in patients with fundic atrophic gastritis. Dig Dis Sci 46: 1573-1583, 2001.

21. Kinoshita Y, Kawanami C, Kishi K, et al. Helicobacter pylori independent chronological change in gastric acid secretion in the Japanese. Gut 41: 452-458, 1997.

22. Sachs G, Meyer-Rosberg K, Scott DR, Melchers K. Acid, protons and Helicobacter pylori. Yale J Biol Med 69: 301-316, 1996.
23. Kato M, Yamaoka Y, Kim JJ, et al. Regional differences in metronidazole resistance and increasing clarithromycin resistance among Helicobacter pylori isolates from Japan. Antimicrob Agents Chemother 44: 2214-2216, 2000.

24. Thung I, Aramin H, Vavinskaya V, et al. Review article: the global emergence of Helicobacter pylori antibiotic resistance. Aliment Pharmacol Ther 43: 514-533, 2016.

25. Asaka M, Sugiyama T, Kato M, et al. A multicenter, double-blind study on triple therapy with lansoprazole, amoxicillin and clarithromycin for eradication of Helicobacter pylori in Japanese peptic ulcer patients. Helicobacter 6: 254-261, 2001.

The Internal Medicine is an Open Access journal distributed under the Creative Commons Attribution-NonCommercial-NoDerivatives 4.0 International License. To view the details of this license, please visit (https://creativecommons.org/licenses/ by-nc-nd/4.0/).

(C) 2019 The Japanese Society of Internal Medicine Intern Med 58: 1549-1555, 2019 